\title{
Discovering, Indexing and Interlinking Information Resources
}

\section{[version 1; peer review: 3 approved with reservations]}

\section{Fabrizio Celli ${ }^{1}$, Johannes Keizer ${ }^{1}$, Yves Jaques ${ }^{1}$, Stasinos Konstantopoulos ${ }^{2}$, Dušan Vudragović 3}

${ }^{1}$ Food and Agriculture Organization of the UN, Rome, Italy

${ }^{2}$ NCSR Demokritos, Athens, Greece

${ }^{3}$ Institute of Physics Belgrade, University of Belgrade, Belgrade, Serbia

V1 First published: $30 \mathrm{Jul} 2015,4: 432$

https://doi.org/10.12688/f1000research.6848.1

Latest published: 17 Nov 2015, 4:432

https://doi.org/10.12688/f1000research.6848.2

\section{Abstract}

The social media revolution is having a dramatic effect on the world of scientific publication. Scientists now publish their research interests, theories and outcomes across numerous channels, including personal blogs and other thematic web spaces where ideas, activities and partial results are discussed. Accordingly, information systems that facilitate access to scientific literature must learn to cope with this valuable and varied data, evolving to make this research easily discoverable and available to end users. In this paper we describe the incremental process of discovering web resources in the domain of agricultural science and technology. Making use of Linked Open Data methodologies, we interlink a wide array of custom-crawled resources with the AGRIS bibliographic database in order to enrich the user experience of the AGRIS website. We also discuss the SemaGrow Stack, a query federation and data integration infrastructure used to estimate the semantic distance between crawled web resources and AGRIS.

Keywords

Linked Data, Text Categorization, Recommender Systems, Web Crawling , AGRIS, SemaGrow

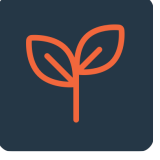

This article is included in the Agriculture, Food and Nutrition gateway.

Open Peer Review
Approval Status
version 2
(revision)
17 Nov 2015
version 1
30 Jul 2015
1. Paolo...............................................................
Newcastle upon Tyne, UK
2. Kei Kurakawa, National Institute of
Informatics, Tokyo, Japan
3. Leonidas Papachristopoulos, Ionian
University, Corfu, Greece
Any reports and responses or comments on the
article can be found at the end of the article.
view


Corresponding author: Fabrizio Celli (fabrizio.celli@fao.org)

Competing interests: No competing interests were disclosed.

Grant information: This work was supported by the European Commission under EU FP7 project SemaGrow (Grant No. 318497), and in part by the Ministry of Education, Science, and Technological Development of the Republic of Serbia (under project ON171017). The funders had no role in study design, data collection and analysis, decision to publish, or preparation of the manuscript.

Copyright: $\odot 2015$ Celli F et al. This is an open access article distributed under the terms of the Creative Commons Attribution License, which permits unrestricted use, distribution, and reproduction in any medium, provided the original work is properly cited. Data associated with the article are available under the terms of the Creative Commons Zero "No rights reserved" data waiver (CC0 1.0 Public domain dedication).

How to cite this article: Celli F, Keizer J, Jaques $Y$ et al. Discovering, Indexing and Interlinking Information Resources [version 1; peer review: 3 approved with reservations] F1000Research 2015, 4:432 https://doi.org/10.12688/f1000research.6848.1

First published: 30 Jul 2015, 4:432 https://doi.org/10.12688/f1000research.6848.1 


\section{Introduction}

AGRIS (http://agris.fao.org/) is the International System for Agricultural Science and Technology, a collection of nearly 8 million multilingual bibliographic resources spanning the last forty years and produced by a network of more than 150 institutions from 65 countries. AGRIS is currently part of the CIARD initiative (http:// www.ciard.net/), a self-described "global movement dedicated to open agricultural knowledge". Some AGRIS data sources are unique (http://aims.fao.org/activity/blog/agris-enriched-data-fao) to the system and AGRIS is the only way in which they can be accessed. The system's goal is to make agricultural research globally discoverable, and as evidenced by Google Analytics it supports both developed and developing countries. Indeed, AGRIS is accessed from more than 200 countries and territories, reaching peaks of 250,000 visits per month. AGRIS users belong to two very different categories: the general public and agriculture professionals. In particular, a survey conducted at the end of 2014 helped to better describe the AGRIS audience [Celli et al., 2015]: researchers, professors, and graduate students looking for bibliographies, librarians, cataloguers, and people responsible for managing and disseminating research outcomes to the community and the rest of the world (including small and big journal publishers), and government officers asking for reports on specific topics. Since December 2013, AGRIS adopted a LOD (Linked Open Data) infrastructure [Anibaldi et al., 2015], which allowed the creation of mashup pages, where users looking for specific topics (e.g. impacts of climate change in a country) can access a publication from the AGRIS database, together with other related resources extracted from other preselected datasets. External resources available in AGRIS mashup pages are not only bibliographic metadata, but also distribution maps, statistics, germplasm accessions, and so on. In this paper we explore a new data source available in AGRIS mashup pages: the web itself.

Nowadays, scientists and researchers publish their results not only in journals or at conferences, but also via web 2.0 tools and other media [Kouper, 2010; Shema et al., 2012] in order to efficiently and broadly communicate their outcomes, a technique that also helps scientific research reach the general public, since newspapers, magazines and science blogs are often the quickest way to reach people informally. Blogs and other websites may also contain a corpus of ongoing research activities, unpublished material, grey literature, quick discussions, and experiments with negative results and ideas. The problem is that this information is usually not exposed using web services that can be consumed by machines, and the only way to access this rich amount of data is to use web search engines that typically return thousands of results, largely meaningless. In addition, most blogs and websites are not well categorized and so it is difficult for users and machines to discover what is actually relevant to the topic of interest.

In this context, we believe that it is important for AGRIS users especially for researchers - to have access to those valuable pieces of information that are neither exposed in a database nor accessible via web service. Our goal is to crawl the web, starting from a list of manually preselected websites and then apply a set of machine learning algorithms to categorize discovered web resources. In recent years, much research has been done to crawl and mine the web. Numerous solutions have been proposed [Liakos et al., 2015; Soulemane et al., 2012; Suganya et al., 2015] to cope with the size of both the publicly indexable and the hidden web employing added semantics to discovered resources and to reuse them in fact sheets and mashups. In fact, it is not only important to discover web links, but also to process them in a way that allows reuse in multiple scenarios. The adoption of ontologies and LOD methodologies helps the analysis and enrichment of discovered web resources [Berendt et al., 2004]. Our work shows how it is possible to apply semantic enrichment to crawled web resources and to use this semantic knowledge to enhance the AGRIS web portal. More specifically, our work leverages Semantic Web technologies and the knowledge encoded in the AGROVOC (http://aims.fao.org/standards/agrovoc/ about) thesaurus in order to recommend web resources that are relevant to a given AGRIS bibliographic item. AGROVOC is a multilingual vocabulary containing more than 32,000 agricultural concepts in 22 languages, aligned with 16 other multilingual knowledge organization systems related to agriculture, and developed by FAO over the course of thirty years [Caracciolo et al., 2011]. Adopting Semantic Web and LOD best practices and technologies, AGROVOC vocabulary items have been assigned URIs, organized into a SKOS-XL concept scheme (http://www.w3.org/TR/skos-reference/skos-xl.html), and served both as Linked Open data and via SPARQL endpoint (http://www.w3.org/TR/sparql11-overview).

In this article we discuss crawling and analyzing web resources to populate our "Crawler Database"; a SPARQL endpoint with AGROVOC annotations of web resources identified by the URL from which they were crawled. By providing web resources with semantics we can use the AGROVOC descriptions of AGRIS bibliographic entries to interlink AGRIS and the Crawler Database. This linking is then exploited by a recommender that identifies web resources that are relevant to AGRIS entries. Furthermore, we also discuss the preliminary testing of the SemaGrow Stack (http://www. semagrow.eu/) as the computational infrastructure for interlinking the AGRIS bibliographic database with the Crawler Database. The query federation and data integration functionalities of the SemaGrow Stack facilitate setting up experiments aiming at estimating semantic similarity between AGRIS entries and other resources. Although the core example that we discuss in this paper is based on the entities described in the Crawler Database, the power of the SemaGrow Stack is that it allows the re-use of this software in the context of different mashup pages combining AGRIS with a variety of LOD sources.

The entire process we discuss in this paper has already been implemented and integrated in the AGRIS website. While the tuning of the recommender system to compute accurate similarities is still an ongoing process, AGRIS mashup pages are enriched with the content of the Crawler Database and statistics are being collected in order to train the recommender system using user behavior. In addition, the workflow and the components described in this paper can be used in any domain, so they are not restricted to agriculture; one can simply use another thesaurus to annotate web resources and populate the Crawler Database. 


\section{Crawling and indexing the web}

The process of discovering and tagging web resources to display new content in the AGRIS website is based on two backend components: a customized Apache Nutch web crawler and AgroTagger. Figure 1 provides an overview of the entire process. As a starting point, in order to display relevant content in AGRIS, we manually preselect related websites to be used as input for the web crawler. Using these URLs the web crawler discovers other related web URLs, while AgroTagger assigns AGROVOC URIs to web URLs and creates the Crawler Database. In sections 2.1 and 2.2 we describe the two backend components.

The web crawler

A web crawler is a piece of software that methodically and automatically analyzes web pages provided as input. Each input web page is a ROOT of the crawling process. During the analysis of a web page, the web crawler discovers all the hyperlinks available in that page, adding them to the list of web pages to be visited. The process stops at a specific depth, i.e. the number of hops a discovered link is from the ROOT. The depth parameter is defined by the user of the web crawler, with the idea that links decrease in relevance as their distance from the ROOT grows. At the end of the crawling process, a list with discovered web URLs is produced. Figure 2 shows an example execution of the web crawler.

In order to implement the process of enriching the AGRIS website with relevant web resources, we used a customized version of Apache Nutch (http://nutch.apache.org/), a highly extensible, scalable and configurable open-source web crawler. Web URLs provided as input were manually selected by experts in the domain of agriculture, in order to start the process from "trusted" and valuable websites. Currently, the depth parameter is set to 5 , since this value seems to be a good compromise between discovering a good quantity of relevant resources and completing the task within an acceptable response time. The application, including the customized Apache Nutch web crawler and some Bash scripts to run it is available on GitHub (https://github.com/fcproj/agrotagger/tree/ master/crawler). The application requires three input parameters:

- The depth of the crawling process

- The path to the directory that stores the output of the process

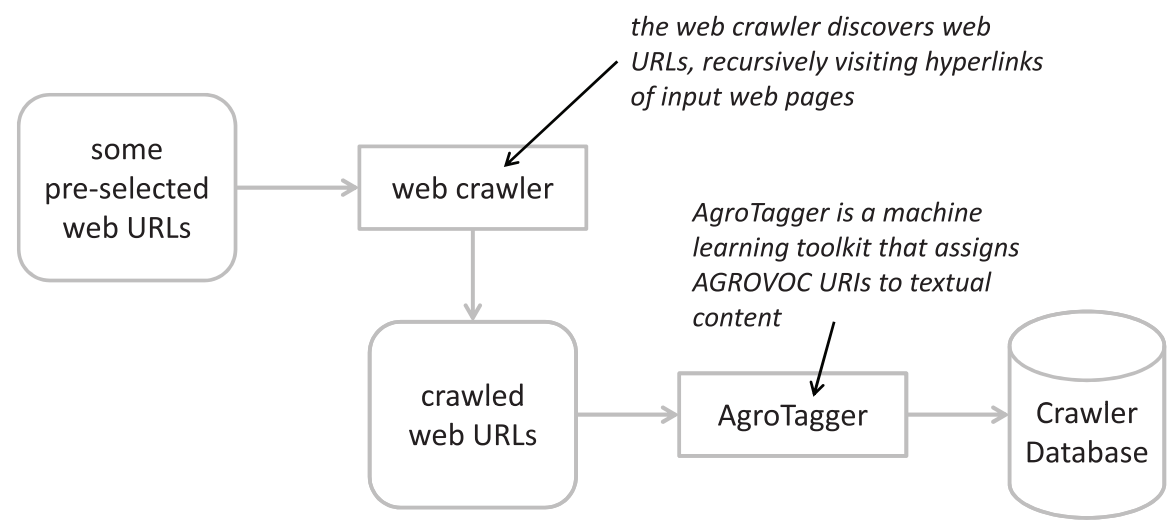

Figure 1. The process of crawling and indexing the web.

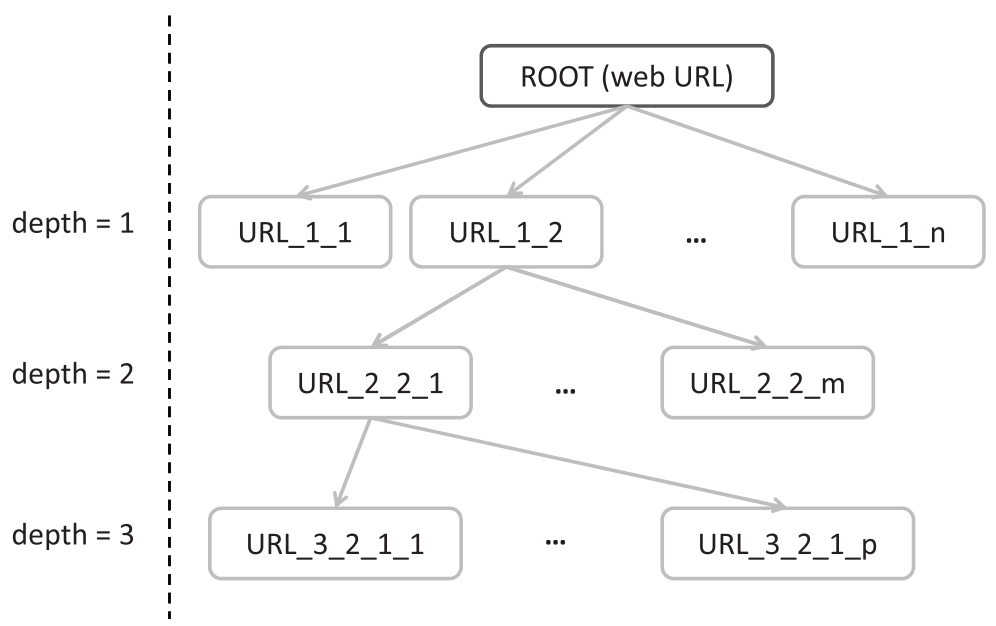

Figure 2. Execution of the web crawler using depth=3. 
- The path to the text file that contains the list of web URLs used as ROOTs by the crawler

The output of the application is a text file containing a structured list of discovered web URLs. Here is a snapshot of the output file:

URL: : http://

URL : : http: / /\%20www. umabroad.umn. edu/students / healthsafety/emergency.php

URL: : http://10-29-2013-tfic-luncheon.eventbrite. $\mathrm{COm} /$

URL : : http: //1z8jbr3nz90837sima2d2fwoktj . wpengine. netdna-cdn. com/wp-content/uploads/2014/05/Nina-Hale-Inc-FactSheet.pdf

URL: : http://2014.northernspark.org/

URL: : http: //2014.northernspark.org/project/ chimera

outlink: toUrl: http://media2.northernspark.org/wp-includes/wlwmanifest.xml anchor:

outlink: toUrl: http://2014.northernspark. org/partners/arts-culture-and-the-creativeeconomy-program-of-the-city-of-minneapolis anchor:

outlink: toUrl: http://2014.northernspark. org/project/bell-museum-staff anchor:

URL: : http://aaea.execinc.com/edibo/

JobMarketCandidates

outlink: toUrl: http://www.aaea.org/ anchor: AAEA

outlink: toUrl: http://aaea.execinc.com/ edibo/LoginHelp anchor: Create an Account / Need Help Logging In

outlink: toUrl: http://www.aaea.org/aboutaaea/aaea-sections anchor: AAEA Sections

\section{AgroTagger classifier}

AgroTagger engine (https://github.com/fcproj/agrotagger) is a toolkit that assigns semantic terms to textual content. At a high level of abstraction, it can be considered as a keyword extractor that uses the AGROVOC thesaurus to extract keywords from a set of web URLs. It is based on MAUI (https://code.google.com/p/mauiindexer), a tool that combines a keyphrase extraction algorithm and a machine learning toolkit for the identification of topics in textual documents. AgroTagger has been trained to assign terms from the AGROVOC thesaurus, even if it currently works only with English documents. Training MAUI with AGROVOC in other languages will allow the applicability of AgroTagger in a multilingual environment, even if further tests need to be performed to understand how the tool behaves with non-Latin characters. Regarding accuracy, a recent test in which AgroTagger results were analyzed by professional indexers showed an accuracy of approximately $80 \%$; in $20 \%$ of the cases the results were too broad. In brief, the accuracy measurement was carried out by domain experts actively involved in the development of the AGROVOC vocabulary based on manual annotations of a test sample from the AGRIS database.
In the process described in this paper, we apply AgroTagger to web URLs discovered by the web crawler and we annotate such URLs with AGROVOC URIs. Annotations are stored in a triple store (the Crawler Database) after which a recommender system defines some relevant combinations between AGRIS bibliographic resources and web documents, making use of AGROVOC as the backbone of the entire process. Considering AgroTagger as a black box, we can describe its I/O as:

- Input: web URLs discovered by the web crawler

- Output: a set of triples that annotate web URLs

AgroTagger is a multi-threaded application, guaranteeing better performance while manipulating web URLs. For each web URL available in the input file, AgroTagger:

- Downloads the resource available at the given web URL and converts it to a text file

- Runs the MAUI indexer trained with AGROVOC

- Produces a set of annotations as RDF triples

Currently, AgroTagger produces an RDF NTRIPLE file that describes semantic annotations for web URLs. Here is an example of an annotated web URL:

<http://www.eje.cz/pdfs/eje/2008/04/01.pdf> <http://www.w3.org/1999/02/22-rdf-syntax-ns\#type> <http://semagrow.eu/rdf\#CrawledDocument > .

<http://www.eje.cz/pdfs/eje/2008/04/01.pdf> <http://purl.org/dc/terms/title> "Word Pro Hoshizaki. lwp" .

<http://www.eje.cz/pdfs/eje/2008/04/01.pdf> <http://purl.org/dc/terms/subject> <http:// aims.fao.org/aos/agrovoc/c_24778> .

<http://www.eje.cz/pdfs/eje/2008/04/01.pdf>

<http://purl.org/dc/terms/subject> <http:// aims.fao.org/aos/agrovoc/c_27496> .

<http://www.eje.cz/pdfs/eje/2008/04/01.pdf> <http://purl.org/dc/terms/subject> <http:// aims.fao.org/aos/agrovoc/c_12332>.

Training AgroTagger with a different thesaurus allows one to reuse the entire workflow and components described in this paper in completely different research domains.

\section{The SemaGrow Stack}

SemaGrow is an FP7 European Project that seeks to develop scalable, efficient, and robust data services needed to take full advantage of the data-intensive and inter-disciplinary Science of 2020, re-shaping the way that data analysis techniques are applied to the heterogeneous data cloud. The core technical outcome of the project is the SemaGrow Stack. The SemaGrow Stack implements a SPARQL endpoint that federates SPARQL endpoints, transparently optimizing federated queries and dynamically integrating heterogeneous 


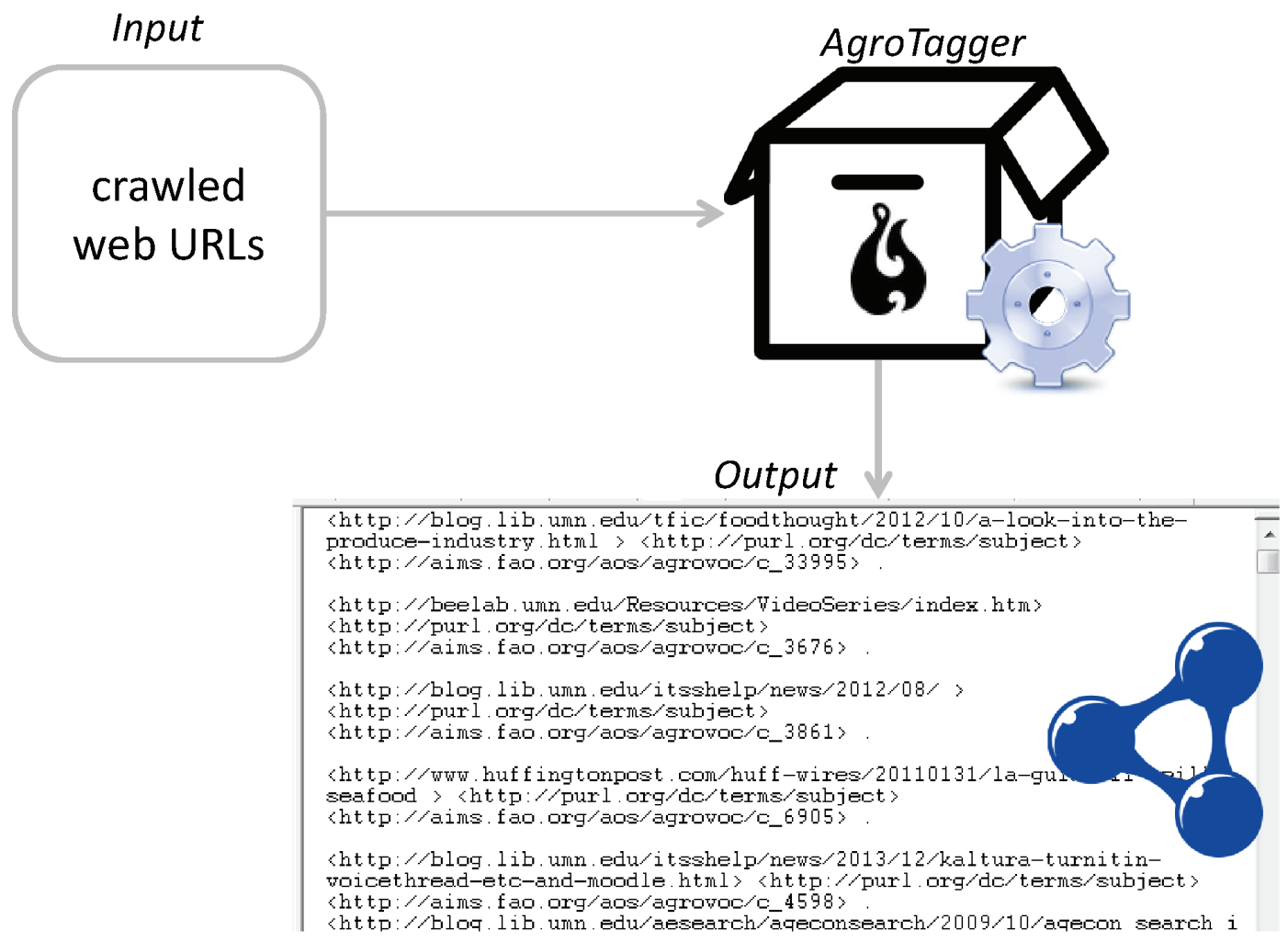

Figure 3. AgroTagger workflow.

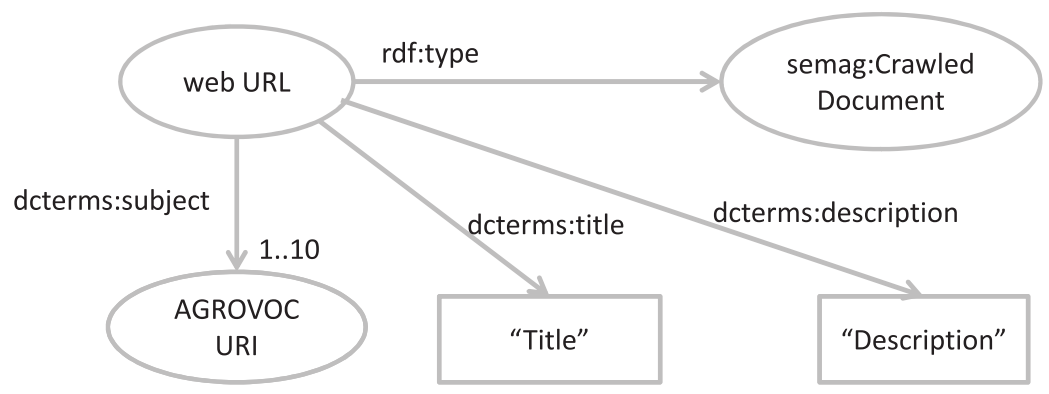

Figure 4. RDF schema of AgroTagger output annotations.

data models by applying the appropriate vocabulary transformations to queries and results [Konstantopoulos et al., 2013].

There are several key features of the SemaGrow Stack that address AGRIS use cases: it provides a querying interface that uses the result of ontology alignment to completely hide schema heterogeneity and also applies methods from database research and artificial intelligence that take into account data contents and optimize federated querying plans. The ontology alignment and dynamic vocabulary transformation facilities allow us to take advantage of multiple agricultural knowledge organization systems that have been aligned to AGROVOC. In this manner, one can develop AGROVOC-aware applications and use them over non-AGROVOC (but aligned) datasets without modification. The query optimizer is based on methods that automatically extract detailed metadata about the content of the federated endpoints, overcoming the lack of detail in manually provided annotations [Zoulis et al., 2015]. As an added benefit, the SemaGrow Stack implements fail-over mechanisms that fall back to alternatives in the face endpoint unavailability. It furthermore does not require any modification of the federated endpoints or any other obtrusion of current workflows. 
The SemaGrow Stack is developed and distributed as open-source software (https://github.com/semagrow) and requires an Apache Tomcat environment (http://tomcat.apache.org/) in order to be deployed and executed.

\section{The recommender system}

The Crawler Database is composed of triples generated by AgroTagger. At this stage, the biggest problem is to compute a meaningful intersection with the AGRIS bibliographic database in order to display relevant information in an AGRIS mashup page. First of all we need to define the concept of "meaningful combinations". A naïve approach is based on counting the number of AGROVOC URIs in common between resources from the Crawler Database and resources from the AGRIS bibliographic database. Thus, for an AGRIS mashup page, we can state that we want to display those resources from the Crawler Database having as many AGROVOC URIs as possible in common with the AGRIS bibliographic entry. To implement this naïve algorithm we developed a recommender system (https://github.com/fcproj/recommender), a JAVA component that computes meaningful combinations between the Crawler Database and the AGRIS database, and generates a new triplestore: the "Recommender Database". The recommender system runs as required as new data is periodically generated by the web crawler.

The recommender system can make use of the SemaGrow Stack as the backbone of the process: in this way, the recommender system is able to compute meaningful combinations between all datasets federated by SemaGrow. Combinations are computed by counting the number of common "dcterms:subject" URIs (http://dublincore. org/documents/dcmi-terms/) between entities of the federated datasets (in the case of the AGRIS dataset, "dcterms:subject" URIs are AGROVOC URIs). The SemaGrow Stack is not strictly necessary, since the recommender system can also work querying two target SPARQL endpoints one by one. In any case, the SemaGrow Stack allows code reuse for all datasets; it is sufficient to configure the SemaGrow Stack by defining the URLs of the SPARQL endpoints included in the federation, and the recommender system can work using a single endpoint. Without the usage of the SemaGrow Stack as an intermediate layer, there is the need to modify the code to add further SPARQL endpoints to the process. Let's explain this statement with an example. Currently, the recommender system has two ways of working (the user can configure it to use either mode): "federated", which requires a single SPARQL endpoint, and "individual", which accepts two SPARQL endpoints. The "federated" mode makes use of the SemaGrow Stack: if the user configures the Stack defining three or four endpoints in the federation, the recommender system can combine all of them. On the contrary, the "individual" mode works with a maximum of two SPARQL endpoints and would require additional software code if one wanted to add further endpoints to the process.

Our experiments focus on the usage of the recommender system to interlink the AGRIS database and the Crawler Database, storing computed combinations in the Recommender Database. At this stage, the algorithm is very easy: we simply need to count the number of common AGROVOC URIs (expressed as objects of a "dct:subject" predicate) between entities of the AGRIS dataset and the Crawler Database. Using the SemaGrow Stack and the single query "federated" mode, the SPARQL query to implement the algorithm is:

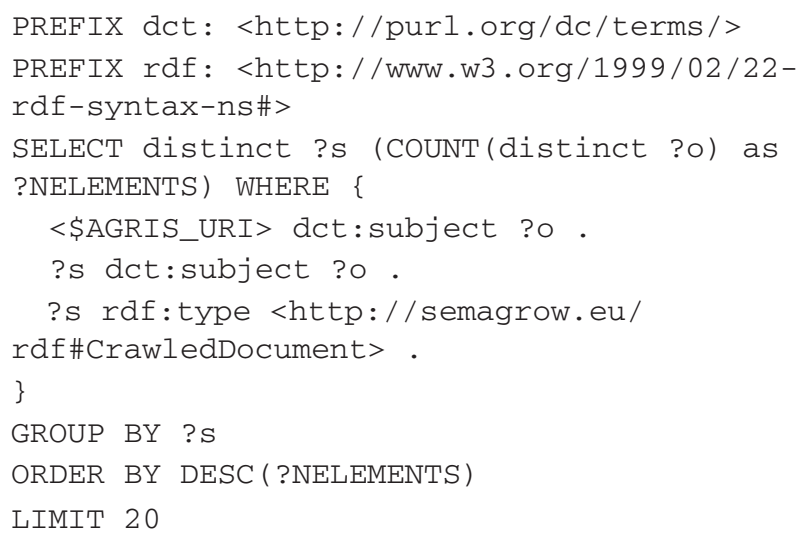

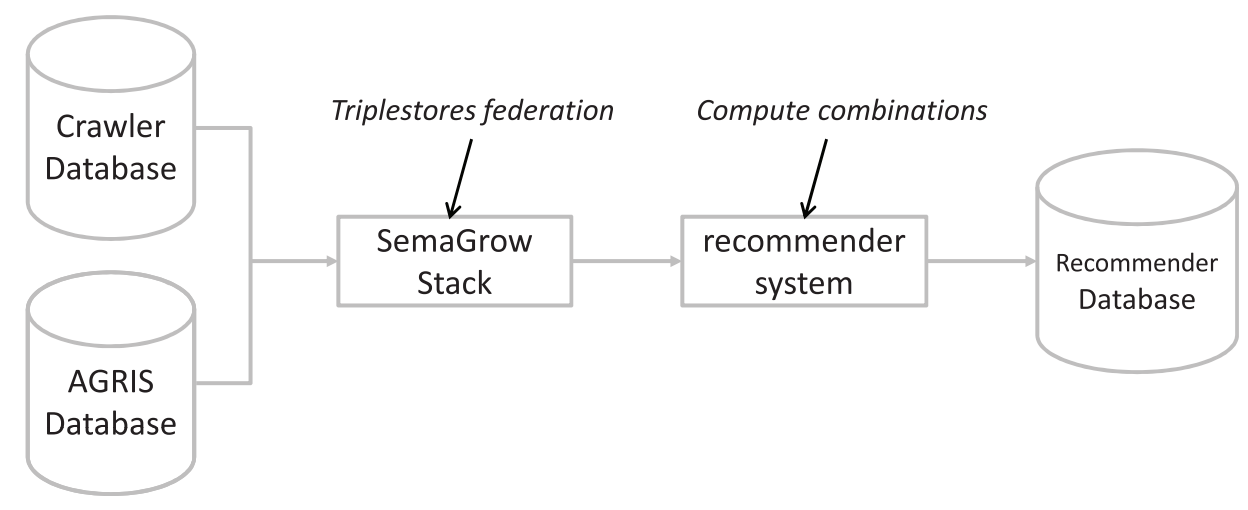

Figure 5. Generation of the Recommender Database, which contains meaningful combinations between the AGRIS bibliographic database and the Crawler Database. 
This SPARQL query sorts resources from the Crawler Database by the number of AGROVOC URIs in common with a given AGRIS entity <\$AGRIS_URI> after which it takes the 20 most relevant web resources and stores them in the Recommender Database as a set of recommendations for an AGRIS URI. In the query, the statement including the $r d f$ : type predicate is only needed in order to get results from the Crawler Database; since the recommender system can work with any dataset federated by the SemaGrow Stack, the type needs to be an application-dependent parameter so that it can be configured to query different datasets.

Unfortunately, there are still few limitations with the SemaGrow Stack. First of all, SPARQL queries must be optimized and they are affected by their content. For instance, using a FILTER statement makes for a high response time. Thus, in order to identify entities from the different datasets federated by the SemaGrow Stack, it is necessary that such datasets define an "rdf:type" (http://www. w3.org/TR/rdf-schema/) for their entities in order to avoid the usage of the FILTER statement. Moreover, given that at the time of these experiments the SemaGrow Stack distribution was limited and allowed only SPARQL queries with dct: subject and/or raf : type predicates, we ran our experiments using the "individual" mode during processing and testing. At the time of publication, our final experiments using the "federated" mode are very promising; SPARQL queries that combine triples from federated datasets show a response time comparable to the execution time of a process that runs individual SPARQL queries to different endpoints and programmatically combines their results.

The "individual" mode that makes use of two different SPARQL queries to two different SPARQL endpoints: AGRIS and the Crawler Database. The first SPARQL query provides the list of AGROVOC URIs (“dct:subject” predicate) for a given $<$ \$AGRIS_URI $>$ :

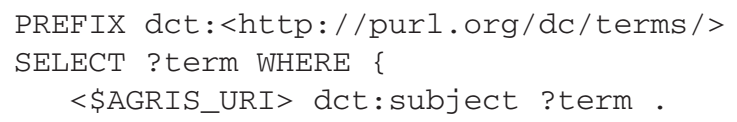

For all AGROVOC URIs provided by the previous query, a second SPARQL query computes the crawled web URLs which contain that AGROVOC URI:

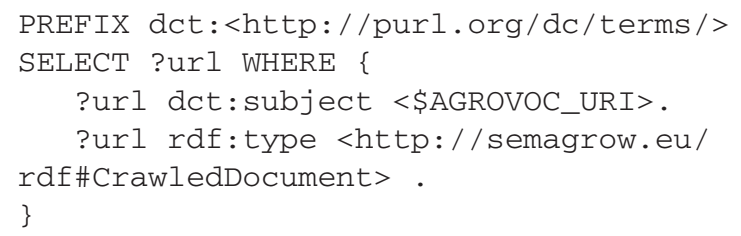

Then, a custom algorithm implemented in JAVA is used to count the number of common AGROVOC URIs between the AGRIS entity and web URLs returned by the previous query, storing the top 20 most relevant web resources in the Recommender Database. The overall custom algorithm can be defined as:

1. Get list "L" of AGROVOC_URIs for a given AGRIS_URI

2. Initialize a Map "MC" whose keys are WEB_ URLs and values are the number of common AGROVOC URIs with the given AGRIS_URI

3. For all AGROVOC_URI "ag" in "L"

a. Get list "WL" of WEB_URLs indexed with "ag"

b. For all WEB_URL "wurl" in "WL"

i. If "wurl" is in "MC", increment the correspondent value

ii. Otherwise, add "wurl" to "MC", setting the value to 1

4. Sort "MC" by values in descending order

5. Take top 20 keys from "MC"

6. Generate recommendations for the AGRIS_ URI

The algorithm was executed on 134,847 AGRIS URIs. The recommender system ran in a CentOS 5 environment with the following configuration:

- Processor: Intel(R) Core(TM) i7 2.80GHz

- Recommender system: 2GB RAM on an 8GB RAM machine, hosted in Rome

- AGRIS SPARQL endpoint: hosted in Malaysia, at MIMOS (http://www.mimos.my/), configuration unknown

- Crawler Database SPARQL endpoint: hosted in Serbia, at IPB (http://www.ipb.ac.rs/index.php/en/), configuration unknown

During the execution of the experiment, the "IPTraf" (http:// iptraf.seul.org/) tool computed a TCP flow rate of between 30 and $45 \mathrm{Kbits} / \mathrm{s}$.

For each AGRIS URI, the computation of combinations required 3.65 seconds on average. The execution time range was between 2.34 and 5.33 seconds for each AGRIS URI. Differences depended on three aspects:

- the number of AGROVOC URIs for a given AGRIS URI, which affects the response time of the AGRIS SPARQL endpoint; 
- the specificity of an AGROVOC concept; broader concepts are associated to many web URLs, so they affect the response time of the Crawler Database SPARQL endpoint and

- network speed and delays.

It is important to remember that this is a background, offline process that runs periodically to keep the dataset up to date. End users querying AGRIS resources will never have to wait for these processes as they have already been performed. Thus, the aforementioned response times are perfectly acceptable.

\section{The similarity index}

In the Recommender Database, recommendations for the same AGRIS URI are sorted by relevance (currently, the relevance is given by the custom algorithm calculated using the number of AGROVOC URIs in common with the AGRIS resource). The recommender system provides a Similarity Score for each recommended web URL, i.e. the percentage of similarity between an AGRIS resource and a recommended web URL. There are three variables to take into account in order to determine the Similarity Score:

- the number of AGROVOC URIs associated with an AGRIS record (we will refer to this variable as \#AGRIS);

- the number of AGROVOC URIs associated with a recommended web URL (\#WEB) and

- the number of common AGROVOC URIs between the web resource and the AGRIS record (\#COMMON).

A naïve approach would consider the Similarity Score as the ratio given by the division between \#COMMON and \#AGRIS:

$$
\text { Score }=\frac{\# C O M M O N}{\# A G R I S}
$$

Unfortunately, this approach has some problems when the AGRIS record is associated with few AGROVOC URIs. For instance, consider the scenario described in Table 1.

Table 1. Example of naïve Similarity Score.
\begin{tabular}{|r|c|c|c|c|c|c|c|}
\hline \#AGRIS & 10 & 10 & 10 & 6 & 6 & 3 & 3 \\
\hline \#WEB & 10 & 10 & 10 & 10 & 10 & 10 & 10 \\
\hline \#COMMON & 10 & 8 & 2 & 6 & 2 & 3 & 1 \\
\hline Naïve Score & 1.0 & 0.8 & 0.2 & 1.0 & 0.33 & $\mathbf{1 . 0}$ & $\mathbf{0 . 3 3}$ \\
\hline
\end{tabular}

The final two scores have an obvious problem of overestimation: the AGRIS record is associated with only 3 AGROVOC URIs and, even if the web URL has 3 common URIs with the AGRIS record, a score of $1.0(100 \%)$ is too much to predict the similarity. Thus, we need an index to adjust the score when the AGRIS record has few AGROVOC URIs associated. This Similarity Index must be 1 in the case of maximum similarity, and 0 in the case of no common AGROVOC URIs. A naïve Similarity Index would be:

$$
S=1-\frac{\# W E B-\# C O M M O N}{\# W E B}
$$

The problem with this index is that \#WEB is equal to 10 in most cases, since AgroTagger assigns 10 AGROVOC URIs to web resources crawled by the web crawler. Thus, $S=1$ only if \#AGRIS is 10 and \#COMMON is 10 , but this situation is highly improbable. If we define a threshold $\tau$ to determine when a number of common AGROVOC URIs is relevant to determine a good Similarity Index, we can improve the quality of the Similarity Score. Currently, we have defined $\tau$ equal to 6 , which means that all cases where \#COMMON is equal or bigger than 6 , the Similarity Index must be 1.0. In this way, defining the correction factor $\mathrm{K}$ :

$$
k=\min (\tau, \# C O M M O N), \text { where } \tau=6
$$

The Similarity Index $\sigma$ can be computed as:

$$
\sigma=1-\frac{\tau-k}{\tau}
$$

This index has all the properties we are looking for: if \#COMMON is $0, \sigma$ is 0 ; if \#COMMON is equals or bigger than the threshold $\tau(6), \sigma$ is 1 . At this point, we can redefine the Similarity Score as:

$$
\text { Score }=\frac{\# C O M M O N}{\# A G R I S} \times \sigma
$$

This approach further improves the Similarity Score. Since \#WEB is never bigger than 10, while \#AGRIS could be ideally any positive integer, we can modify the denominator of the first factor introducing the upper limit $T$, which is the maximum number of AGROVOC URIs associated to an AGRIS resource that is meaningful to the computation of the Similarity Score (we define $T=10$ ):

$$
\text { Score }=\frac{\# C O M M O N}{\min (T, \# A G R I S)} \times \sigma=\frac{\# C O M M O N}{\min (T, \# A G R I S)} \times 1-\frac{\tau-\min (\tau, \# C O M M O N)}{\tau}
$$

Where: $T=10$ and $\tau=6$.

Table 2 revises the scenario described in Table 1, computing the similarity score with the last formula.

Table 2. Revised example of Similarity Score.
\begin{tabular}{|r|c|c|c|c|c|c|c|}
\hline \#AGRIS & 10 & 10 & 10 & 6 & 6 & 3 & 3 \\
\hline \#WEB & 10 & 10 & 10 & 10 & 10 & 10 & 10 \\
\hline \#COMMON & 10 & 8 & 2 & 6 & 2 & 3 & 1 \\
\hline Naïve Score & 1.0 & 0.8 & 0.2 & 1.0 & 0.33 & 1.0 & 0.33 \\
\hline Real Score & 1.0 & 0.8 & $\mathbf{0 . 0 7}$ & 1.0 & $\mathbf{0 . 1 1}$ & $\mathbf{0 . 5}$ & $\mathbf{0 . 0 6}$ \\
\hline
\end{tabular}


The output of the recommender system

Currently, the recommender system provides RDF in the RDF/ XML serialization. As an example:

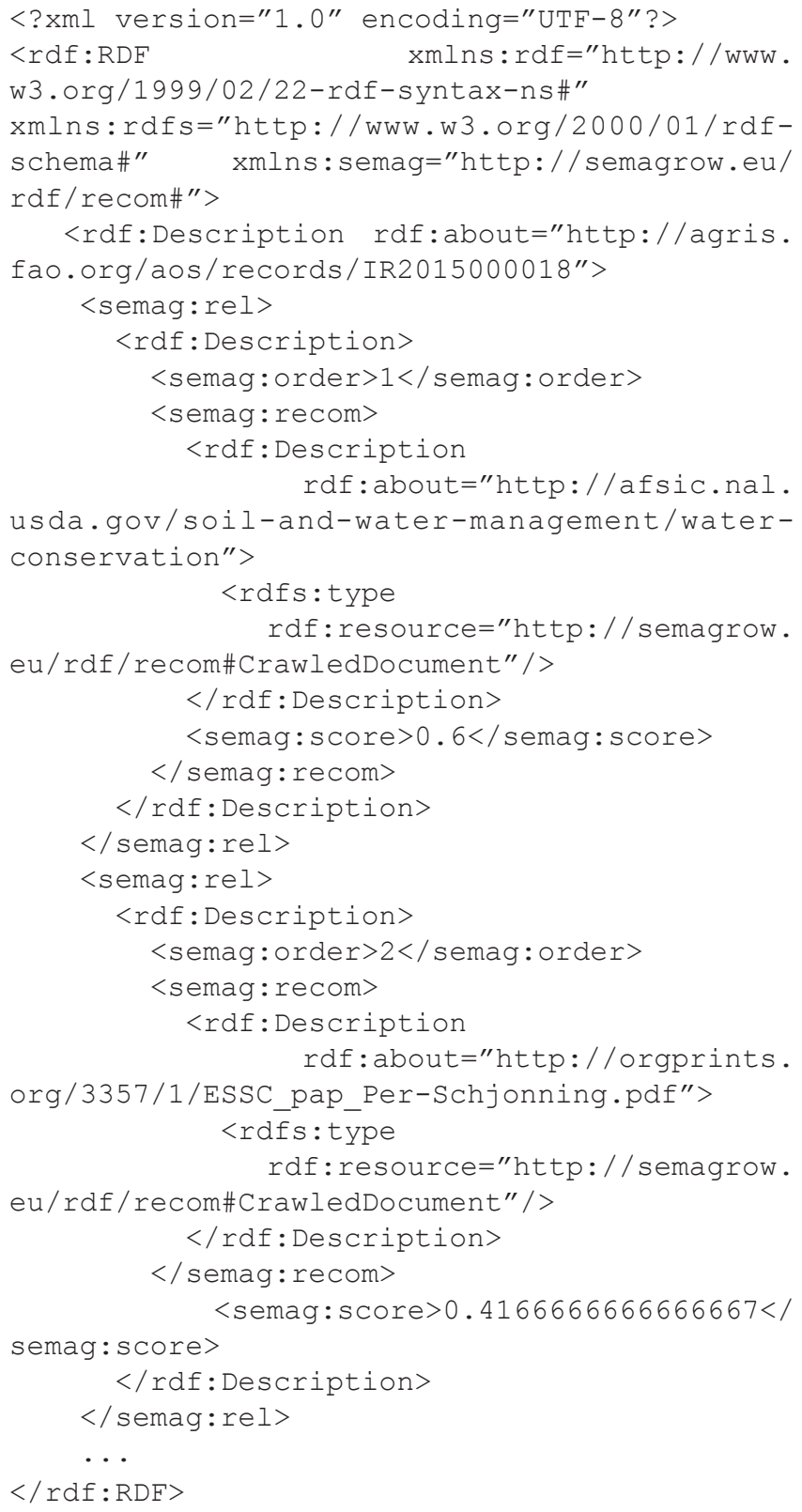

The custom algorithm to compute meaningful combinations is an area for further research and improvement. In particular, other parameters may play a key role to define the relevance of a web resource. For instance, the system might check if some AGROVOC terms used by an AGRIS record appear in the title of the crawled web resource, or in its description. Then, AgroTagger could also be improved to return the ranking score of each AGROVOC URI assigned to a web resource, in order to use such a score in the algorithm that computes combinations. In addition to that, we can observe users' behavior in an AGRIS mashup page in order to assign more relevance to web resources more frequently clicked by AGRIS users. Furthermore, interviews of users will be conducted to evaluate the relevance of resources displayed in AGRIS mashup pages. Finally, there may be other more exotic algorithms to calculate similarity that can give greater relevance to end users, but such experiments were outside the scope of this project.

\section{The AGRIS front-end}

The creation of an AGRIS mashup page is a dynamic and sensitive process, which is made possible by the usage of AGROVOC as the backbone of the system. In fact, AGRIS records come with AGROVOC URIs and, relying on AGROVOC formal alignments to many thesauri, it is possible to query publicly accessible web services or SRARQL endpoints to provide access to resources indexed with various thesauri. Thus, when the user selects a publication from the AGRIS database, the system can display related information on the same topic. External data sources are identified based on the content, the relevancy to the AGRIS domain, and the information provider [Celli et al., 2015].

This paper presented a set of components that implement a process of discovering web resources related to AGRIS records in order to enrich the user experience in AGRIS mashup pages. We also ran experiments with the SemaGrow Stack as a backend component that can easily extend the data sources federated by the AGRIS mashup pages. These experiments led directly to the addition of a new data source to the AGRIS mashup pages: the dataset of related resources crawled from the web.

The creation of this new data source required setting up a process that used numerous components. We implemented an automatic process that makes use of a web crawler to discover web resources, and relies on AgroTagger to annotate discovered URLs with AGROVOC URIs. Then, in order to compute meaningful combinations between the AGRIS database and the Crawler Database, we implemented a recommender system to define the relevance of web resources. The output of this process is a widget in the AGRIS mashup pages that, reading the content of the Recommender Database (that is continuously updated by an offline process), can display relevant resources from the web. In this way, we believe that AGRIS users may find relevant data that assists them in working with agricultural issues and food security.

\section{Qualitative analysis of relevance: an example}

In this section we show a recommendation example in order to start a brief discussion about the relevance of resources contained in the widget of Figure 7. Our starting point is the AGRIS article "Water Conservation of Qanat, using Optimum use of Water in Unused Seasons (Case study: Dehraz Qanat of Sabzevar)" (http://agris. fao.org/agris-search/search.do?recordID=IR2015000018). This article exposes some criteria to optimize the use of water carried by qanats (a qanat is an underground channel to transport water from an aquifer under a hill, especially for irrigation of hot and arid environments). Thus, as it is highlighted by the title, the article is mainly about "water conservation". It has been indexed with eleven AGROVOC terms, including water management, soil sciences, soil conservation, and water conservation. The recommender system 


Q

\author{
A About
}

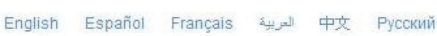

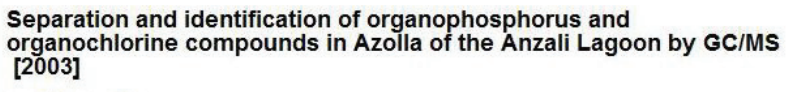

\title{
RDF L Iod: :ive
}

Sayyadnejad, Mohammad Ali, Islamic Azad University, North Tehran Branch Amini Ranjbar, Gholamreza, Islamic Azad University, North Tehran Branch

Abstract:

In this research sampling of Azolla was done in the southwest (Siahkeshim), center (Hendekhaleh) and east (Sheijan) of the Anzali Lagoon for two seasons (Summer and Autumn 2001). Extraction of organophosphorus and organochlorine compounds in Azolla were done by mixture of methylene chloride- acetone solvents. Separation and identification of compounds in extracted samples were done by GC-NPD, ion trap and quadrupole GC/MS Sequentially. The most important pollutant identified in Azolla was Fenitrothion pesticide. Selected lon Monitoring (SIM) technique was also used for further confirmation of Fenitrothion and the quantitative analysis was done by GC-NPD. The highest concentration of Fenitrothion in Azolla on wet weight quantitative analysis was done by GC-NPD. The highest concentration of Fenitrothion in Azolla on wet weight
basis was $591 \mathrm{ng} / \mathrm{g}$ in the southwest region and the lowest concentration $341 \mathrm{ng} / \mathrm{g}$ in the east region of Lagoon in Summer. Keywords : Azolla, Anzali Lagoon, GCMS, Organophosphorous and organochlorine compounds

\begin{tabular}{|c|}
\hline - Agrovoc Keywords \\
\hline $\begin{array}{l}\text { : pollutants } \\
\text { : organochlorine compounds } \\
\text { : organophosphorus compounds } \\
\text { plant protection } \\
\text { Azolla } \\
\text { : pesticides } \\
\text { : lagoons } \\
\text { - } \text { traxic substances } \\
\text { Irlamic Republic }\end{array}$ \\
\hline - Other information \\
\hline Volume: 16 \\
\hline Issue: 3 \\
\hline Extent: pp: $14-21$ \\
\hline Language: per \\
\hline Type: Journal Article \\
\hline $\begin{array}{l}\text { Other subjects: Fenitrothion pesticide: } \\
\text { Anzali Lagoon; GC/MS }\end{array}$ \\
\hline
\end{tabular}

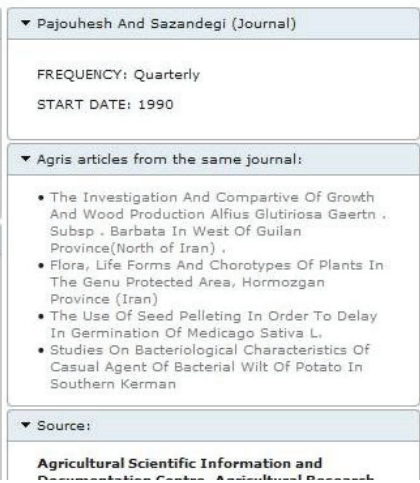

Figure 6. An AGRIS mashup page displaying an AGRIS bibliographic record with related information from external data sources.

suggests five web resources related to this article, with a similarity score ranging between $42 \%$ and $60 \%$. Such web resources are available in the widget "Activities from the web".

A manual analysis of results across a sample of AGRIS records by FAO consultants indicated that a Similarity Score bigger than 50\% for results showed a good level of precision. In fact, in the example we are analyzing, the first suggestion is a web page of the USDATA. GOV website (http://afsic.nal.usda.gov/soil-and-water-management/water-conservation); the score is $60 \%$ and the resource is completely related with the article, since it contains other resources about "water conservation" coming from USA universities and other agencies. Matching AGROVOC terms of the AGRIS article with the title of this resource would have further increased the Similarity Score, since the title itself is about "water conservation". Regarding the remaining four suggestions, the example shows how a similarity score of $42 \%$ does not always predict good results. For instance, the web page of Cornell University (http://conservationagriculture. mannlib.cornell.edu/pages/resources/photosvideos.html) is relevant to the article, since it contains - among other things - some Power Point presentations about "water management" and "soil management". Conversely, the other resources with the same similarity score of $42 \%$ are irrelevant. As previously discussed, improving the recommender system by matching AGROVOC terms of the AGRIS article with the titles and descriptions of crawled web resources should greatly improve the quality of results: in this example, the

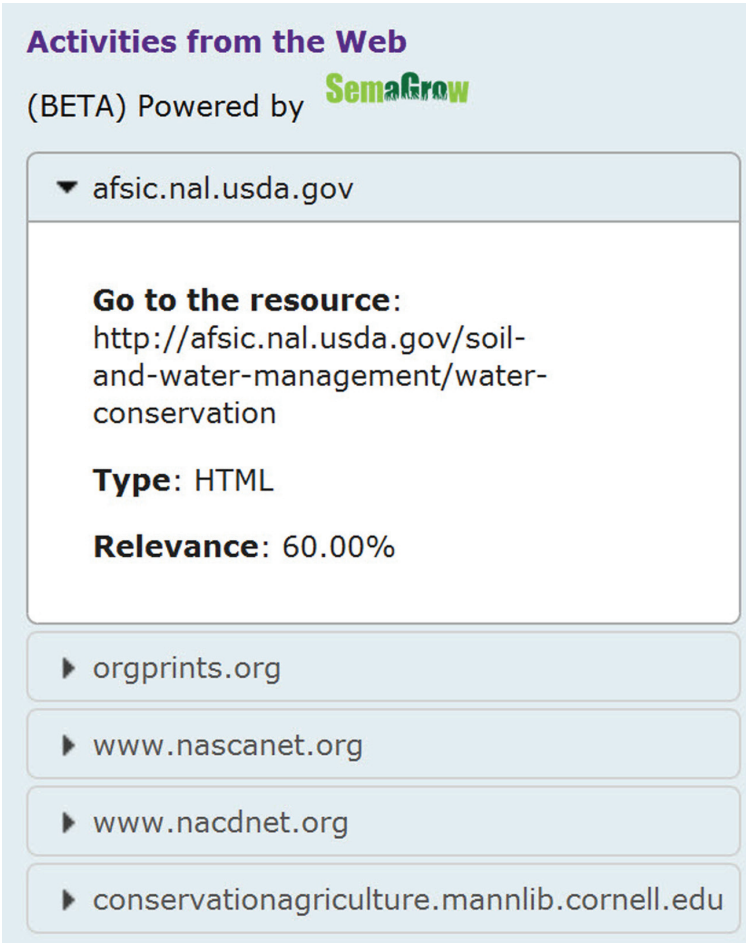

Figure 7. A widget displaying related resources from the web. 
improved algorithm would have suggested the XML feeds from EPRINTS about "agricultural water management", or the PDF document entitled "Agricultural Perspectives on Water Resource Management in the Americas", both of which are quite relevant and yet missing from the current list.

\section{Conclusions}

The web contains much latent knowledge, especially when that knowledge is expressed as unstructured and poorly categorized full-text content. This paper describes a proposed solution to discover such knowledge making use of modified open source software (Nutch and Maui) together with the SemaGrow Stack and a custom recommender in order to enrich the relevance of AGRIS bibliographic resources and hence the AGRIS web portal mashup.

The adoption of the SemaGrow Stack as a backend facilitated the development of a recommender engine as it was possible to implement the system without requiring any prior knowledge of the specifics of the datasets that are combined with the AGRIS database. In this manner, the system can be re-used with any dataset using AGROVOC (or any terminology aligned to AGROVOC) to describe websites, experiments, software, or any resources relevant to agriculture. We are now able to show to AGRIS users any relevant content extracted from the web, something possible thanks to the adoption of semantic web technologies. The entire process will continue to be extended and improved as experiments continue: AgroTagger and the recommender system will be tuned to guarantee better precision in the computation of recommendations (for instance, trying to match AGROVOC keywords with titles of target resources and experimenting with alternative algorithms), while the SemaGrow Stack will be optimized and used to federate additional data sources.

Furthermore, as previously mentioned, training AgroTagger with a different thesaurus allows to apply the entire process to different research domains, and not only to the AGRIS website.

\section{Software availability}

Latest source code (AgroTagger): https://github.com/fcproj/agrotagger

Latest source code (recommender system): https://github.com/ fcproj/recommender

Archived source code at the time of publication (AgroTagger): http://dx.doi.org/10.5281/zenodo.20777 (Celli, 2015a).
Archived source code at the time of publication (recommender system): http://dx.doi.org/10.5281/zenodo.20775 (Celli, 2015b).

License: CC BY 4.0 http://creativecommons.org/licenses/by/4.0/

Author contributions

FC conceived conceptual ideas, implemented all the software components (except for the SemaGrow Stack), designed the experiments, and prepared the manuscript.

JK made provided guidance to the development of the AGRIS website, as well as conceptual suggestions to the implementation of the crawling and tagging processes.

YJ participated in AGRIS modelling, study design and selection of technology solutions. He also prepared and revised the language of the manuscript.

SK contributed sections 3 and part of section 1 . He was also responsible for the conceptual and technical development of the SemaGrow Stack.

DV supported the testing of the crawling and recommendation processes, providing access to computing resources of PARADOX supercomputing facility at the Scientific Computing Laboratory of the Institute of Physics Belgrade.

All authors were involved in the revision of the draft manuscript and have agreed to the final content.

\section{Competing interests}

No competing interests were disclosed.

Grant information

This work was supported by the European Commission under EU FP7 project SemaGrow (Grant No. 318497), and in part by the Ministry of Education, Science, and Technological Development of the Republic of Serbia (under project ON171017).

I confirm that the funders had no role in study design, data collection and analysis, decision to publish, or preparation of the manuscript.

\section{Acknowledgments}

We acknowledge use of computing resources of PARADOX supercomputing facility at the Scientific Computing Laboratory of the Institute of Physics Belgrade. 
Anibaldi S, Jaques $Y$, Celli F, et al:: Migrating bibliographic datasets to the Semantic web: The AGRIS case. Semantic Web. 2015; 6(2): 113-120. Publisher Full Text

Berendt $B$, Hotho A, Mladenic $D$, et al.: A roadmap for web mining: from web to semantic web. Web Mining: From Web to Semantic Web. vol. 3209 of Lecture Notes in Computer Science, Springer, Berlin, Germany. 2004; 3209: 1-22. Publisher Full Text

Caracciolo C, Morshed A, Stellato A, et al.: Thesaurus Maintenance, Alignment and Publication as Linked Data: the AGROOVOC use case. In Proceedings of the 5th Intl Conference on Metadata and Semantic Research Proceedings, Izmir, Turkey. 2011; 240: 489-499.

Publisher Full Text

Celli F, Malapela T, Wegner K, et al:: AGRIS: providing access to agricultural research data exploiting open data on the web [v1; ref status: approved 1 http://f1000r.es/599]. F1000Res. 2015; 4: 110

Publisher Full Text

Celli F: agrotagger: crawler agrotagger 125 DOI. Zenodo. 2015a. Data Source

Celli F: recommender: agris_recommender_system_1_3_2_DOI. Zenodo. 2015b. Data Source

Konstantopoulos S, Koukourikos A, Karampiperis P: Improving the Real-time Performance of Heterogeneous Extremely Large Datasets. In Proc. of the 17th
Panhellenic Conference on Informatics, Thessaloniki, Greece. 2013; 287-293. Publisher Full Text

Kouper I: Science blogs and public engagement with science: Practices, challenges and opportunities. Journal of Science Communication. 2010.

Reference Source

Liakos $\mathrm{P}$, Ntoulas A, Labrinidis A, et al.: Focused crawling for the hidden web. In Proceedings of the World Wide Web 2015 Conference Proceedings, Florence, Italy. 2015. Publisher Full Text

Shema H, Bar-Ilan J, Thelwall M: Research Blogs and the Discussion of Scholarly Information. PLOS ONE. 2012; 7(5): e35869.

Publisher Full Text

Soulemane M, Rafiuzzaman M, Mahmud H: Crawling the Hidden web Approach to Dynamic web Indexing. International Journal of Computer Applications. 2012; 55(1): 7-15.

Publisher Full Text

Suganya Devi R, Manjula D, Siddharth RK: An Efficient Approach for

Web Indexing of Big Data through Hyperlinks in Web Crawling.

ScientificWorldJournal. 2015; 2015: 739286.

PubMed Abstract | Publisher Full Text | Free Full Text

Zoulis N, Mavroudi E, Lykoura A, et al.: Workload-Aware Self-Tuning Histograms of String Data. In Proceedings of the 26th DEXA Conference (DEXA 2015), 1-4 September, Valencia, Spain. 2015. 


\section{Open Peer Review}

\section{Current Peer Review Status: ? ? ?}

\section{Version 1}

Reviewer Report 12 October 2015

https://doi.org/10.5256/f1000research.7363.r10769

(c) 2015 Papachristopoulos L. This is an open access peer review report distributed under the terms of the Creative Commons Attribution License, which permits unrestricted use, distribution, and reproduction in any medium, provided the original work is properly cited.

\section{Leonidas Papachristopoulos}

Department of Archives, Library Science and Museology, Ionian University, Corfu, Greece

Current study entitled "Discovering, Indexing and Interlinking Information Resources" consists of a technical evaluation of an enriching system attached to an agricultural bibliographic database called AGRIS. I am not sure about the novelty of the system that is presented but I can admit that is an interesting work which does not pose any reading difficulties to the reader, even to those who are not so familiar. Lately, recommending systems have been set on the center of the research activity and the specific work is following the research trends of the area.

In my opinion there are some gaps in the study. For example on the description of the Agrotagger crawler researchers say:

"Regarding accuracy, a recent test in which AgroTagger results were analyzed by professional indexers showed an accuracy of approximately $80 \%$; in $20 \%$ of the cases the results were too broad. In brief, the accuracy measurement was carried out by domain experts actively involved in the development of the AGROVOC vocabulary based on manual annotations of a test sample from the AGRIS database"

It is not clear to me if the aforementioned test has been held in the context of another previous study or it covers the specific study. If it has been held in the past a reference is necessary. Otherwise researchers need to provide further details. Additionally I think that authors should provide more information about accuracy tests. In my opinion the paper describes adequately the design and implementation but needs further enrichment regarding evaluation data.

Authors should consider papers restructuring as in many cases I get lost between implementation and evaluation points.

Some minor syntax comments are:

In Page 1: "AGRIS database, together with other related resources extracted from other preselected datasets"... "together with" should be changed to "combined with". 
In Page 1: "Nowadays, scientists and researchers publish their results not only in journals or at conferences, but also via web 2.0 tools and other media [Kouper, 2010; Shema et al., 2012] in order to efficiently and broadly communicate their outcomes, [a technique that also helps scientific research reach the general public, since newspapers, magazines and science blogs are often the quickest way to reach people informally]." For better understanding the sentence should be split into two. From the point "a technique' should start another sentence.

In Page 1: "Blogs and other websites may also contain a corpus of ongoing ..." "Corpus" should be changed into "corpora"

In Page 2: "Adopting Semantic Web and LOD best practices and technologies, AGROVOC vocabulary items have been assigned URIs, organized into a SKOS-XL concept scheme (http://WwW.W3.org/TR/skosreference/skos-Xl.html), and served both as Linked Open data and via SPARQL endpoint ( http://WWW.w3.org/TR/sparq/11-overview)." Re-write the sentence for better understanding.

Competing Interests: No competing interests were disclosed.

\section{I confirm that I have read this submission and believe that I have an appropriate level of expertise to confirm that it is of an acceptable scientific standard, however I have significant reservations, as outlined above.}

Author Response 03 Nov 2015

Fabrizio Celli, Food and Agriculture Organization of the United Nations, Italy

Really thanks for your comments. As you suggested, the "AgroTagger" section was improved. We added a reference to a paper including details about the MAUI training set. We also added a more precise description of the test sample used to measure accuracy of AgroTagger. In addition to that, evaluation of performance was moved to a separate section, together with further details as the comparison between the execution time in the individual and federated modes. Moreover, we improved the evaluation of relevance of recommendations: we ran an analysis of relevance on a test set of resources in the domain of fisheries, computing precision and providing other comments. We hope that, based on these modifications, the referee can now fully approve our article.

Competing Interests: No competing interests were disclosed.

Reviewer Report 28 September 2015

https://doi.org/10.5256/f1000research.7363.r10291

(c) 2015 Kurakawa K. This is an open access peer review report distributed under the terms of the Creative Commons Attribution License, which permits unrestricted use, distribution, and reproduction in any medium, provided the original work is properly cited. 


\section{Kei Kurakawa}

National Institute of Informatics, Tokyo, Japan

This paper proposed a functional enhancement for the agricultural journal article bibliographic database search, AGRIS, whose bibliographic page has additional web resources information relevant to its content. The web resources are automatically crawled by a customized Nutch web crawler, and labeled with some agricultural index terms, i.e. AGROVOC by a kind of machine learning module, the AgroTagger classifier. Since AGRIS bibliographies are already labeled with AGROVOC, the paper proposed a similarity measure between AGRIS bibliographies and web resources based on the number of common and originally labeled AGROVOC terms on them. AGRIS bibliographies and web resources are stored in SPARQL servers, so that the SemaGrow stack is used to easily send queries to them to calculate the similarity scores. The recommender system provides at most top 20 relevant web resources for an AGRIS bibliography by the calculation, which is serialized on RDF/XML.

I recognize that the whole framework to construct the new functionality is valuable, but the paper is not sufficient with computational performance evaluation on the framework and quality evaluation for the linkages between bibliographies and web resources in an approved scientific method.

Below are the suggestions for minor improvements.

In page 3:

In the "Crawling and indexing the web" section, the author describes "In the section 2.1 and 2.2" in the leading paragraph. The section numbers might not be forbidden to point to sections of this article.

In page 4:

In the left column of the "web crawler" sub-section, the author shows a snap shot of the output file of a Nutch web clawer. Please describe the structure of the file. I would like to know what some tags mean to construct the structure. Why is a blank URL listed in the example?

In the left column of the "AggroTagger classifier" sub-section, the author mentions the quality of tagging by AgroTagger such as "AgroTagger results were analyzed by professional indexers showed an accuracy of approximately $80 \%$; in $20 \%$ of the cases the results were too broad." I would like to know more precise descriptions of the quality of tagging. How large is the training set? How did the author construct the training set? Please describe the training set. In addition, the test set for tagging and examining its accuracy should be described.

In the "AggroTagger classifier" sub-section, the figure 3 and 4 should be explicitly referenced. In the current article, those figures are not referenced in any sentences.

In the first phrase of "The SemaGrow Stack" section, the author refers "the data-intensive and inter-disciplinary Science of 2020". Does "2020" means "Horizon 2020"? Or, does it refer to another funding framework? Science of 2020? If it is another funding framework, please cite a relevant web site for it. Please make sure what it is. 
In page 6:

In the "recommender system" section, the second paragraph of "The recommender system can make use of ... add further endpoints to the process", the figure 5 should be explicitly referenced. In addition, for good understanding of reconfigurable structures consisting of the "federated" and the "individual", the author had better depict both structures in the figure 5 .

In page 7:

In the right column, the author describes "the overall custom algorithm" as a list form. For better understanding, this algorithm should be represented in a pseudo-programming code form.

In the right column, the author mentions some experimental conditions and results of the number of AGRIS URIs and the average execution time. In addition, how many AGROVOC URIs are prepared for AGRIS URIs or Web URLs in this experiment? How long does it take for all processing? Please figure out the time of processing in the "individual" mode and the "federated" mode. Which is better from the viewpoint of computation?

In page 8:

In the right column, line 13, "factor K" should be changed to the lower case "factor k".

In the last similarity formula, "braces ( )" should be written in the proper place.

In table 2, "Real Score" might be " Revised Score"?

In page 9:

In the right column, the section "AGRIS front-end", please indicate explicitly figure 6 in a sentence.

Competing Interests: No competing interests were disclosed.

\section{I confirm that I have read this submission and believe that I have an appropriate level of expertise to confirm that it is of an acceptable scientific standard, however I have significant reservations, as outlined above.}

Author Response 03 Nov 2015

Fabrizio Celli, Food and Agriculture Organization of the United Nations, Italy

We would really like to thank Dr. Kei Kurakawa for this useful review. The paper was improved following his comments. In particular:

1. We separated the performance experiments from the deployment section. Performance experiments were performed again using a bigger test set and we compared execution time in the "individual" mode with the execution time in the "federated" mode.

2. We ran an analysis of relevance on a test set of resources in the domain of fisheries. We were able to compute precision of linkages, adding some additional details.

3. The "web crawler" section was revised: we described the structure of the output file, which is generated using the "readseg-dump" command line tool provided by Apache Nutch. The blank URL listed in the example was meaningless, so we removed it. 
4. The "AgroTagger" section was revised too, with the addition of a more precise description of the test sample used to measure accuracy. Then, the paper including the work of training MAUI was cited.

5. In the "SemaGrow Stack" section, the reference to the "Science of 2020" was removed, since it was misleading. In fact, it is part of SemaGrow proposal description, so it does not explicitly refer to Horizon 2020 or to any other framework, but to a general view of the world in 2020.

6. All minor improvements suggested by the reviewer were implemented.

We hope that, based on these modifications, the referee can now fully approve our article.

Competing Interests: No competing interests were disclosed.

Reviewer Report 24 August 2015

https://doi.org/10.5256/f1000research.7363.r9730

(C) 2015 Missier P. This is an open access peer review report distributed under the terms of the Creative Commons Attribution License, which permits unrestricted use, distribution, and reproduction in any medium, provided the original work is properly cited.

\section{Paolo Missier}

School of Computing Science, Newcastle University, Newcastle upon Tyne, UK

Overall, I found the article interesting and easy to read. In line with the F1000 reviewers' guidelines, I am going to comment "on the quality of the research and whether the article is scientifically sound (i.e. whether the work has been well designed, executed and discussed)", rather than on novelty, also making suggestions for improvements in the presentation.

The work partly builds on prior established tools designed for the AGRIS system, mainly the AGROVOC thesaurus, as well as methods for federating SPARQL queries over heterogeneous resources (the SemaGRow stack).

The original research contribution consists mainly of a focused crawler that looks for web resources that are relevant to the AGRIS community, the AgroTagger annotation tool, which annotates those web resources with semantic terms from the AGROVOC thesaurus, and a recommender tool that uses the annotated database to suggest web resources that are likely to be relevant to a given AGRIS bibliographic item.

The annotation model is fine, however the evaluation part of the paper is weak, especially with regards to recommendation. This is where I think the authors have an obligation to report on the accuracy (ie F-scores) of their method on a benchmark of test cases, especially since substantial effort is spent in the paper to explain the similarity score used by the recommender. This is completely missing, however, the only evidence of the method at work being an example towards the end. For an experimental paper, this is hardly acceptable. So my main substantial recommendation is to strengthen the evaluation section (this may appear in other articles cited in 
the references, however that was not immediately clear to me).

On matters of form and presentation, I found that the methods, including the recommender algorithm, are very simple (by the authors' own admission) and thus in my opinion the exposition can be greatly abbreviated. For example, fig. 2 and 3 are not very informative and could be removed. Also the lengthy RDF fragments are not very significant (but if you need them, please switch to $n 3$ notation!), the annotation model of fig 4 is very simple (essentially, just a dcterms:subject), the 6-points algorithm is a straightforward intersection operation and has already been described in the text, the queries on pg 7 are fairly simple, etc.

Further minor presentation suggestions:

You may not need to build up the similarity score from naive to final, possibly just describe the final version?

There is a mix of design and implementation / performance considerations, these may be best kept separate.

A new section header may be missing after the first paragaph of "agris front end"?

Competing Interests: No competing interests were disclosed.

I confirm that I have read this submission and believe that I have an appropriate level of expertise to confirm that it is of an acceptable scientific standard, however I have significant reservations, as outlined above.

Author Response 03 Nov 2015

Fabrizio Celli, Food and Agriculture Organization of the United Nations, Italy

We would really like to thank Dr. Paolo Missier for his useful comments.

We ran an analysis of relevance on a test set of resources in the domain of fisheries. We were able to compute precision, but not recall. In fact, recall is mainly related to the web crawling, and it is not easy to estimate it. In addition to that, in the context of the AGRIS website it is not strongly important: we are more interested in a good precision, especially because we display only 5 recommendations per AGRIS resource.

We separated the performance experiments from the deployment section, adding further details. Performance experiments were performed again using a bigger test set and we compared execution time in the "individual" mode with the execution time in the "federated" mode.

We abbreviated a bit the exposition (for instance, we removed the six-points algorithm and the RDF fragment related to the output of the recommender system), but we maintained the discussion about the flow of thoughts leading from the naïve similarity score to the final one because we thought it helps in justifying some parameters available in the final formula.

We hope that, based on these modifications, the referee can now fully approve our article. 
Competing Interests: No competing interests were disclosed.

The benefits of publishing with F1000Research:

- Your article is published within days, with no editorial bias

- You can publish traditional articles, null/negative results, case reports, data notes and more

- The peer review process is transparent and collaborative

- Your article is indexed in PubMed after passing peer review

- Dedicated customer support at every stage

For pre-submission enquiries, contact research@f1000.com 